\title{
Vínculo prenatal: la importancia de los estilos vinculares en el cuidado gestacional ${ }^{1}$
}

Prenatal Attachment: the importance of Attachment Styles in the prenatal care.

Vínculo pré-natal: a importância dos estilos de vinculação no cuidado pré-natal

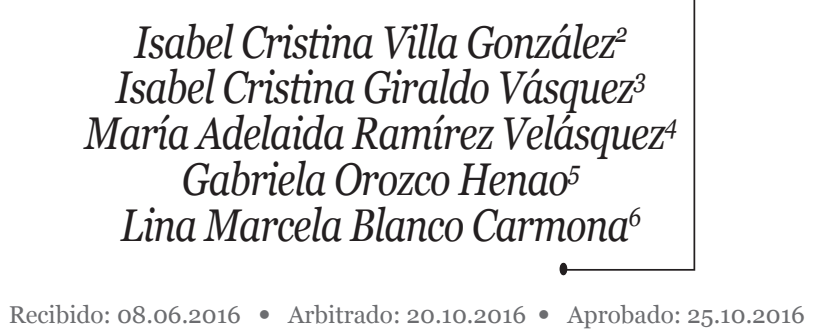

${ }^{1} \mathrm{El}$ presente artículo corresponde a la revisión bibliográfica y el análisis documental sobre el vínculo prenatal de una investigación en curso denominada: Factores Biológicos Psicológicos y Socioculturales de los Hábitos Saludables en Pacientes Gestantes Atendidas en una Institución de Salud de Alta Complejidad de la Ciudad de Medellín, desarrollada por un equipo interdisciplinario de 8 investigadores pertenecientes a 6 grupos de investigación de la Universidad Pontificia Bolivariana de Medellín - Colombia, 2015.

${ }^{2}$ Psicóloga, Magister en Psicología, Especialista en Familia, Docente Titular Facultad de Psicología, Investigadora grupo ECCO, Escuela de Ciencias Sociales, Universidad Pontificia Bolivariana, Medellín (Colombia), Isabel. villa@upb.edu.co.

${ }^{3}$ Auxiliar de investigación, estudiante de pregrado de la Facultad de Psicología, Universidad Pontificia Bolivariana, Medellín (Colombia). isabelc.giraldo@ upb.edu.co

${ }^{4}$ Auxiliar de investigación, estudiante de pregrado de la Facultad de Psicología, Universidad Pontificia Bolivariana, Medellín (Colombia), mariaadelaida. ramirez@upb.edu.co

${ }^{5}$ Auxiliar de investigación, estudiante de pregrado de la Facultad de Psicología, Universidad Pontificia Bolivariana, Medellín (Colombia), gabriela.orozco@upb.edu.co

${ }^{6}$ Auxiliar de investigación, estudiante de pregrado de la Facultad de Psicología, Universidad Pontificia Bolivariana, Medellín (Colombia), lina.blanco@upb.edu.co 


\section{Resumen}

El presente artículo de revisión busca identificar antecedentes y aproximaciones teóricas contemporáneas sobre el tema del 'vínculo afectivo' o 'estilo vincular personal' y su relación con el 'vínculo prenatal' que establecen las mujeres en estado de embarazo con sus bebés en gestación. Así mismo, identificar aspectos relevantes sobre esta relación y los cuidados de la salud gestacional, que sirvan de sustento a la innovación pedagógica de las acciones en salud. Es un estudio cualitativo de investigación documental. El desarrollo del tema se realizó a través de un proceso inductivo en el que la información se organizó en un sistema categorial en tres fases: exploración, focalización y profundización y que dio lugar a los subtemas desarrollados, que partieron de los constructos de apego y vínculo desde los autores originales. Esta revisión posibilita la reflexión sobre las actividades educativas tradicionales del modelo biomédico y sugiere mejores prácticas en los programas de atención prenatal que favorezcan, desde acciones colectivas e individuales con las gestantes, espacios y experiencias formativas que posibiliten cambios en las conductas de salud para ellas y sus bebés en desarrollo. Se propone tomar en consideración en la atención prenatal, todas las acciones que favorezcan un vínculo seguro y estable y garantice la unidad de cuidado del binomio madre-hijo como protector natural de los bebés por nacer.

Palabras clave: vínculo afectivo, embarazo, estilo de vida, autocuidado.

\section{Abstract}

This review article aims to identify antecedents and contemporary theoretical approaches on the issue of bonding or link style staff and their relationship to the established link prenatal women who are pregnant with their unborn babies. Also, identify relevant aspects of this relationship, and gestational health care, to serve as support to the pedagogical innovation of health actions. It is a qualitative study of documentary research. The development of the subject was carried out 
through an inductive process in which information was organized into a categorical system in three phases: exploration, targeting and deepening and which gave rise to the developed sub-themes, which started from attachment and bond constructs from the original authors. This revision allows reflection on traditional educational activities of the biomedical model and suggests best practices in prenatal care programs that favor from collective and individual actions with pregnant women, spaces and formative experiences that enable changes in health behaviors for themselves and their developing babies. It is proposed to consider in prenatal care, all activities fostering a safe and stable relationship and ensure care unit of the mother-child as a natural protector of unborn babies.

Keywords: bonding, pregnancy, lifestyle, self-care.

\section{Resumo}

O presente artigo de revisão busca identificar antecedentes e aproximações teóricas contemporâneas sobre o tema do vínculo afetivo ou estilo de vinculação pessoal e sua relação com o vínculo pré-natal que as mulheres em estado de gravidez estabelecem com seus bebês em gestação. Da mesma forma, identificar aspectos relevantes sobre esta relação e os cuidados da saúde gestacional, que sirvam de apoio à inovação pedagógica das ações em saúde. É um estudo qualitativo de pesquisa documental. O desenvolvimento do tema foi realizado através de um processo indutivo, no qual a informação foi organizada em um sistema categorial em três fases: exploração, focalização e aprofundamento, e que deu lugar aos subtemas desenvolvidos, os quais partiram das construções sobre apego e vínculo a partir dos autores originais. Esta revisão possibilita uma reflexão sobre as atividades educativas tradicionais do modelo biomédico e sugere melhores práticas nos programas de atenção pré-natal que favoreçam, a partir de ações coletivas e individuais com as gestantes, espaços e experiências de formação que possibilitem mudanças nas condutas de saúde para elas e seus bebês em desenvolvimento. Propõe-se levar em consideração na assistência 
pré-natal, todas as ações que favoreçam um vínculo seguro e estável e que garantam a unidade a unidade do cuidado do binômio mãe-filho com protetor natural dos bebês próximos a nascer.

Palavras chave: bonding ligação, gravidez, estilo de vida, auto-cuidado

\section{Introducción}

El presente artículo tiene como propósito identificar algunas aproximaciones teóricas acerca del 'estilo vincular de las mujeres' y su relación con 'el vínculo prenatal' que establecen con sus bebés en gestación. A su vez, con esta revisión, se pretende reflexionar acerca de las acciones de profesionales de las Ciencias Sociales y de la Salud respecto a cómo potencializar desde la atención primaria, a través de los programas de educación en salud gestacional, la vinculación sana de las gestantes con sus bebés por nacer, a partir de la reflexión subjetiva e intersubjetiva de sus propias experiencias y relaciones, y así contribuir a mejorar las condiciones socio afectivas en las que estos bebés nacen, como estrategia de protección social a la diada madre/hijo y al desarrollo de las futuras generaciones.

Las investigaciones acerca de las repercusiones que las relaciones afectivas tempranas generan en el desarrollo psicológico de los seres humanos, se han convertido en una prolífera línea de indagación científica y una generosa fuente de evidencia para la psicología del desarrollo contemporánea. Los resultados investigativos han permitido comprender, desde una perspectiva evolutiva y evolucionista, el peso que tienen la calidad de la interacción afectiva y la cualidad de los cuidados tempranos en la construcción de la identidad personal de los seres humanos, tanto en el desarrollo infantil como en la vida en general, pues representan el soporte relacional primario en la construcción de los recursos cognitivos y afectivos con los que cuentan los individuos para afrontar la vida (Blair-Gómez, 2013). Como se explicará más adelante, las condiciones y cualidades en las que dicho vínculo temprano se desarrolla, se constituye en la base emo- 
cional sobre la cual, desde el punto de vista relacional, se posibilita la consolidación de personalidades más sanas y resistentes o, por el contrario, más vulnerables a presentar dificultades psicológicas con los riesgos que conllevan las psicopatologías emocionales asociadas (Grimalt y Heresi, 2012). Las teorías clásicas de finales de siglo XIX, desde diferentes orillas del conocimiento científico, comenzaron enfatizando en la importancia de una adecuada relación de cuidado y protección de las crías humanas con sus cuidadores primarios desde el momento del nacimiento y, tradicionalmente, han definido precisamente, este momento como el tiempo o a partir del cual los dispositivos biológicos de especie, modulados por las dimensiones afectivas, sociales y culturales, 'gatillan' o disparan una suerte de comportamientos de cuidado hacia el bebé recién nacido, que son garantes de la supervivencia de este, quien a su vez, cuenta en su incipiente e inmadura estructura biofísica, con dispositivos biológicos similares a los de los adultos para responder a ese cuidado y 'conectarse' con su(s) cuidador(es), para con él constituir una diada que garantice la supervivencia y el desarrollo sano de la cría.

En el contexto actual, las 'teorías del vínculo' se pueden considerar un estudio interdisciplinario, puesto que tienen en cuenta postulados de las teorías clásicas del psicoanálisis y de las relaciones objetales, hasta los avances en neurodesarrollo de las Ciencias Cognitivas contemporáneas y las teorías evolutivas, etológicas y psicológicas más actuales.

Siendo la madre biológica, la cuidadora natural del bebé en la mayoría de las especies mamíferas y en la nuestra, en épocas recientes el interés se ha focalizado en identificar las condiciones y características que desde la gestación, o antes de esta, posibilitan la emergencia de este sistema básico de cuidado al momento del nacimiento, como dispositivo primario y previo a la vinculación del nuevo miembro de la sociedad con el mundo de sus congéneres y con la vida misma. Esta revisión de tema da cuenta de algunos de los constructos que son fundamento de las teorías del vínculo y su importancia en la psicoevolución humana, y se centran en el vínculo prenatal como condi- 
ción previa a esta vinculación afectiva y su relación con los cuidados gestacionales. El propósito del presente escrito es identificar en estos postulados oportunidades para revisar las prácticas tradicionales de educación para la salud de los programas de atención primaria en población gestante respecto a la inclusión de estrategias pedagógicas centradas en los elementos afectivos tanto de la personalidad materna, como de la relación prenatal con el hijo por nacer.

\section{Metodología}

Es un estudio cualitativo de tipo documental. El desarrollo del tema se realizó a través de un proceso inductivo en el que la información se organizó en un sistema categorial en tres fases exploración, focalización y profundización y que dio lugar a los subtemas desarrollados, que partieron de los constructos de apego y vínculo desde los autores originales. Luego se expone con amplitud la categoría de vínculo prenatal y, finalmente, la relación de este constructo con la calidad de vida, la salud y los factores protectores del desarrollo pre y postnatal de los niños. Los criterios de selección del material consistieron en el rastreo de las bases teóricas sobre la teoría del vínculo, sin límite de fecha, y posteriormente, las investigaciones y desarrollos teóricos (basados en evidencia empírica) de los últimos 20 años acerca de la importancia del vínculo afectivo en la etapa prenatal del desarrollo y su relación con la salud gestacional.

Las técnicas de recolección de datos y almacenamiento de información utilizadas fueron: el rastreo en las bases de datos Ebsco, Science Direct, Pubmed y Scielo y la elaboración de RAE (Resúmenes Analíticos Especializados) para artículos científicos y fichas de contenido para libros, capítulos de libro, tesis doctorales y otros documentos académicos. La sistematización de la bibliografía y la organización de las diversas teorías, ideas y fuentes de información rastreadas, se realizó con el fin de facilitar el trabajo posterior de análisis y redacción. 


\section{Resultados}

\section{El concepto de vínculo y su importancia para las teorías del desarrollo humano}

Para Oiberman (1998, 2001), vínculo, del latín vinculum (atadura), representa una conexión estrecha, pero no forzada, entre varios sujetos que implica proximidad, y que nace de la voluntad, inicialmente del niño, como un aspecto innato que está en función de su preservación, pero también del adulto cuidador que experimenta un interés genuino de protección y apoyo. Según el principal exponente de esta teoría, Bowlby (1989), se define como «cualquier forma de conducta que tiene como resultado el logro o la conservación de la proximidad con otro individuo claramente identificado al que se le considera mejor capacitado para enfrentarse al mundo» (p.40). Para este autor, la función biológica de dicha conducta de apego es de protección y se caracteriza por la intensidad de la emoción que lo acompaña, y de esta depende el tipo de relación que se establecerá entre el apegado y la figura de apego.

Bowlby (1986) es uno de los principales precursores de las teorías del vínculo; para él siempre ha existido la necesidad universal humana de formar vínculos afectivos. Su interés por formular esta teoría inició con la observación de niños perturbados emocionalmente por estar separados de sus familias. Principalmente trabajó con niños que tenían problemas de conducta, descubriendo que las experiencias de la infancia determinan el desarrollo de la personalidad en cuanto al establecimiento de lazos afectivos.

Por otro lado, Safram y Segal (1994) entienden por vínculo afectivo «el lazo emocional que desarrolla el niño con sus padres o cuidadores y que le proporciona la seguridad emocional indispensable para el desarrollo de sus habilidades psicológicas y sociales» (p. 45). Bowlby (1989) da cuenta de la existencia del sistema de apego como un sistema filogenéticamente heredado y presente en los seres humanos. El autor define: 
Es un sistema comportamental innato, propio de los seres humanos, que se activa en situaciones de amenaza o aflicción y que tiene la finalidad de recuperar el bienestar, a través de comportamientos destinados a recibir apoyo, cuidado y protección del cuidador primario. El apego conforma un sistema de regulación diádica entre el cuidador y el infante, siendo imprescindible la presencia y disponibilidad del cuidador, ya que dependiendo de las experiencias repetidas (positivas, negativas o ambas), el niño desarrolla una serie de representaciones mentales acerca de la naturaleza de la relación con su figura de apego y acerca de su propia existencia (p. 242).

En las últimas décadas, las teorías evolucionistas del desarrollo desde la Escuela Cognitivo- Constructivista y más específicamente, el enfoque Postracionalista, retoman las teorías del vínculo como el marco explicativo integrador del desarrollo temprano. Guidano (1994), uno de los representantes de esta corriente teórica, define «el conocimiento como la manera en que el organismo organiza sus relaciones con lo externo» (p. 3). Para el autor, la realidad equivale a la manera en que se conoce y se concibe el mundo; donde el amor, que se rige por la emoción, es el organizador de dicho conocimiento y, por tanto, de esta realidad.

El concepto del amor, en la acepción de relación y vínculo afectivo, también ha sido trabajado por Maturana (2008), quien lo define como un fenómeno que se construye a partir de la relación entre los seres humanos. Esta emoción implica una serie de conductas donde se concibe la existencia del otro como un ser diferente a sí mismo, con significados personales, en la cercanía de las relaciones que se establecen y a partir de las cuales el ser humano sólo puede conocerse en relación con los otros, viéndose en ellos, lo que se denomina realidad intersubjetiva. Dicha realidad, según Guidano (1994), es producto de un proceso evolutivo que, al ponerse en práctica, posibilita pautas espacio-temporales estables, dónde la relación de apego temprana es la unidad sobre la que se fundamenta y se constituye la base para la posterior formación del self.

Guidano planteó además que las relaciones de apego son fundamentales para la construcción de un sentido de un Sí mismo, estable y continuo en el tiempo, puesto que se trata de un proceso autorreferen- 
cial. Afirma: «en los seres humanos, como en todos los organismos vivientes, el sistema afectivo emocional corresponde a una aprehensión inmediata e irrefutable del mundo» (2008, p.90). Respecto al lenguaje, este crea las condiciones para el incremento de un sentido de identidad, definido como 'mismidad' que va aumentando las posibilidades de interacción y vinculación afectiva que cada vez se complejizan en el entramado relacional de la vida social de cada individuo (Guidano, 1994; Balay, 1994).

Si bien el vínculo busca mantener durante el desarrollo proximidad y cercanía, también busca afianzar la identidad personal. Lecannelier (2009) citando a Trevarthen (1979) nos plantea que, antes del nacimiento y en los primeros años de vida, existen ciertas conexiones interpersonales que comprueban que, desde la evolución, la intersubjetividad hace parte del desarrollo humano, a lo que se le denomina intersubjetividad primaria. El niño desde los primeros días de vida muestra una sintonía y una preferencia exclusiva hacia los sonidos, olores y las expresiones faciales de la madre.

Para Lecannelier (2010), el apego es la matriz general donde opera una dinámica de acercamiento/alejamiento en el vínculo hacia los otros. Dentro de esta, ocurre un encuentro entre dos personas en el que se dan procesos intersubjetivos, por esto la intersubjetividad constituye un principio explicativo ordenador del desarrollo, que le permite al niño mantener su coherencia e integridad a lo largo de su proceso evolutivo. Esto se logra «sobre la base de una conexión y sintonía emocional con los otros, quienes constituyen una referencia» (Lecannelier, 2009, p.8). En este sentido, Bowlby (1986) afirma que en el ser humano existe una disposición a crear lazos emocionales íntimos con individuos determinados como un componente básico de la naturaleza humana; esta interacción es equivalente a la calidad del vínculo, que el autor define como:

El conjunto de pautas de conductas características, en parte preprogramadas, que se desarrollan en el entorno corriente durante los primeros meses de vida y que tienen el efecto de mantener al niño en 
una proximidad más o menos estrecha con su figura materna (Bowlby, 1989, p. 15).

El sentimiento y la conducta de la madre hacia su bebé están influenciados por las experiencias personales de apego pasadas y la relación establecida con sus padres. Bowlby (1989) lo sustenta, entre otras evidencias, en las investigaciones con padres que fueron maltratados por sus progenitores y que posteriormente maltratan a sus hijos, desde un principio de auto perpetuación de sus modelos vinculares primarios.

La calidad del apego temprano, depende en mayor medida de un cuidador siempre accesible y receptivo, es decir disponible, para que el niño experimente los primeros sentimientos positivos, seguridad, afecto y confianza, en lugar de los negativos, inseguridad, abandono o miedo. Además, debe contar con la sensibilidad suficiente para entender las peticiones del bebé y así poder atender sus necesidades, logrando una interacción cooperativa.

A partir de su investigación y un importante trabajo con población clínica y comunidad general, Bolwby (1989) describe tres (3) principales pautas de apego: Apego seguro, donde el niño cuenta con unos cuidadores accesibles, sensibles y colaboradores, por lo tanto se atreve a explorar el mundo; Apego ansioso resistente, en el que el niño no está seguro sobre dichas cualidades de su progenitor, por lo tanto, no siente que éste lo ayudará cuando sea necesario, es decir tiende a la separación ansiosa, al aferramiento y es ansioso para explorar el mundo; Apego ansioso elusivo, en el que la persona cuando busca cuidados espera ser despreciado en vez de amparado, porque sufrió del rechazo de la madre cuando buscó protección y la Versión desorganizada de las pautas típicas, donde el individuo se muestra desorientado, desorganizado y puede presentar algunas de las pautas anteriores pero en una versión más caótica y desorganizada, generalmente presente en niños que han sido maltratados y/o descuidados de manera extrema y reiterativa por los padres (Bowlby 1989). 
Estos patrones de interacción con los padres son el punto de partida desde el cual los infantes construyen modelos internos interpersonales (denominados de trabajo) a partir de los cuales construyen su mundo interpersonal y desarrollan representaciones mentales o modelos internos de referencia para relacionarse con otros y que, como explica Brandon (2006), toman forma de «expectativas, respuestas e interpretaciones de la conducta interpersonal» (p. 1). Estos modelos internos de trabajo se refieren al conjunto de tendencias comportamentales particulares en las relaciones que se establecen como íntimas, así mismo están involucradas con las representaciones y las interacciones padres-niño después de su nacimiento (Maas, Vreeswijk, Cock \& Rijk, 2012).

Por su parte, desde las escuelas cognitivas en psicología, la terapia centrada en los esquemas propuesta por el Dr. Jeffrey Young, en 1999 (TCE), integra los importantes aportes de la teoría del apego y amplía los aportes del modelo de la Terapia Cognitiva tradicional de Aaron Beck, centrada en la explicación de la personalidad y de los problemas psicológicos a partir del concepto de esquema disfuncional (unidades de conocimientos, creencias y emociones disfuncionales y negativos). El concepto central de dicha terapia lo constituyen los esquermas maladaltativos tempranos o EMT y son el núcleo fundamental de sus intervenciones clínicas en pacientes con patología severa. Los EMTs se definen como amplios patrones que comprometen a los recuerdos, sensaciones corporales, emociones y cogniciones que el sujeto posee acerca de sí mismo y acerca de sus relaciones con otros (Young, 1999). Se desarrollan en las primeras interacciones con los padres, cuidadores y pares; es decir, su cuna son las relaciones significativas tempranas enmarcadas en el estilo vincular familiar, y constituyen el núcleo de temas que incluyen al sí mismo, las normas y límites sociales y las relaciones con los demás. Los EMTs comprenden elementos que fueron adaptativos, pero luego interferidos por las connotaciones afectivas negativas cuya rigidez e inflexibilidad se convierten en estrategias disfuncionales en la adolescencia y la adultez, en especial, en lo que se refiere al establecimiento de relaciones afectivas significativas cómo el amor de pareja, las relaciones de cuidado, cómo la diada madre- hijo y demás relaciones significativas. 
La clasificación de los EMTs propuesta por Young incluye 18 EDTs que se agrupan en 5 dominios o dimensiones que corresponden a las relaciones tempranas disfuncionales relacionadas con los estilos de apego inseguro de tipo evitante o ambivalente antes descritos y al tipo de necesidades no contempladas durante la infancia y que dan origen a los EMTs del adulto. Los dominios de dichos esquemas son: desconexión y rechazo, pobre autonomía y desempeño, límites insuficientes, orientación hacia los otros y finalmente, el dominio hipervigilancia e inhibición (Young, 2008). En cierto modo los modelos internos de trabajo antes descritos sobre sí mismo y los otros, podrían ser similares a los modelos de esquemas, debido a que reflejan las representaciones cognitivas de las relaciones que han sido generalizadas a través de las interacciones con figuras importantes en la temprana infancia del individuo, e incluso influyen en los pensamientos y creencias acerca de las relaciones con otros importantes. En el caso, por ejemplo, de individuos que configuren un apego inseguro el funcionamiento y organización de los modelos de trabajo conduce a percepciones distorcionadas respecto a las interacciones interpersonales y de esta forma propian escenarios para mantender relaciones disfuncionales con otros (Valdizan, 2003).

Como puede verse en esta teoría clínica, las cualidades y características del vínculo temprano influyen de manera significativa en los sistemas de creencias sobre sí mismo y los demás y por tanto, en los estilos de personalidad adulta (Cordero \& Tiscornia, 2000). Estos planteamientos teóricos tienen implicaciones en el desarrollo respecto a la madre gestante y su capacidad para cuidar y amar a su hijo desde antes del parto, así como la manera en la cual el bebé construya la representación de sí mismo y de los demás, a partir de las cualidades del vínculo temprano con su madre.

A manera de síntesis de lo expuesto hasta este momento, se dirá que, el vínculo es un proceso psicológico, por esto el sistema de apego está ligado a la construcción de un sentido de sí mismo, y la manera como la persona ve a los otros y al mundo (Guidano, 2001), es decir, el estilo vincular es el medio por el cual se logra establecer un sentido de 
sí mismo específico, único y continuo en el tiempo, por medio del cual el sujeto logra reconocerse. Es así como para este autor y la corriente psicológica que representa, el desarrollo del vínculo se da simultáneamente con el desarrollo de la identidad, que es referenciada inicialmente por la percepción de los otros y, después auto referenciada y mantenida desde una identidad personal.

Aunque siempre se ha dicho que la función última del apego es la protección del infante ante cualquier peligro potencial, Blair-Gómez (2013) realiza un interesante rastreo que evidencia cómo las funciones del apego trascienden la dimensión efectiva y están implicadas en el desarrollo integral, mostrando su potencial para explicar el funcionamiento posterior del niño. Algunos de los estudios citados por la autora son: el desarrollo de esquemas o percepciones del mundo, el bienestar emocional del niño (Volling, McElwain, Notaro \& Herrera, 2002; Waters et al., 2010), su salud psicológica (Anglin, Cohen \& Chen, 2008; Brumariu \& Kerns, 2008), y la determinación de la calidad de las relaciones posteriores con los demás (Levy \& Kelly, 2010), incluyendo la forma en la que ella o él actuarán como padres en el fututo (Van Ijzendoorn, 1995; Grienenberger, Kelly \& Slade, 2005), citados en Blair-Gómez (2013).

El apego es un proceso de auto-referencia que le permite al individuo construir un sentido consistente, estable y continuo de sí mismo (Guidano, 1999). Desde este principio evolutivo Blair-Gómez (2013) afirma que:

En su inmensa y profunda necesidad de sobrevivir, un niño siempre va a desarrollar una relación de apego con su cuidador (es), sin importar cuales son las características de sus cuidadores. El resultado de la relación y del desarrollo socio-emocional de niño, va a depender en gran medida en las características de cuidador, básicamente su disponibilidad emocional, su sensibilidad emocional y a la respuesta a las necesidades y los signos de malestar del niño (p. 36).

A la organización del estado intersubjetivo, definido como el paquete de emociones básicas del ser humano, que forman una organización unitaria de todas las tonalidades emocionales, se conoce como sentido 
personal, que es la manera específica de sentirse en el mundo (Guidano, 2001).

Puede deducirse entonces, que el apego se inicia desde el mismo momento en que la madre se 'vincula' con su hijo y su condición de madre y esto ocurre desde el momento que conoce su estado de embarazo, y prosigue durante la vida adulta y la vejez. Este proceso se conoce como apego prenatal, que según Eswi \& Khalil (2012), es el vínculo de la madre con su feto entendiéndose como un proceso durante el cual, las mujeres embarazadas experimentan sentimientos y emociones por su bebé, interactúan con él y desarrollan una identidad materna, que se evidencia principalmente, en las representaciones maternas que la futura madre hace de su hijo y que interviene en la manera como esta interactúa, fantasea y cuida de su estado de gestación. Esta relación en el binomio madre-hijo que comienza a establecerse antes del nacimiento, se ve influenciada por variables afectivas, sociales, culturales de diversa naturaleza, además del acceso y la calidad de los servicios de atención en salud de la gestante; algunas de las cuales serán revisadas a continuación.

\section{El Vínculo Prenatal}

A partir del conocimiento del estado de embarazo, una mujer tiene nueve meses para asimilar la idea de tener un bebé y se va conectando poco a poco con él, mientras su hijo está en el vientre, tiene tiempo de imaginarlo y representarlo, sin embargo, cuando el bebé nace la madre tiene que ajustar el conjunto de representaciones con la realidad.

Para Bowlby (Laxton-Kane \& Slade, 2002) el apego prenatal es la relación que se da entre la madre y su hijo antes de nacer, que hace emerger sentimientos y emociones por su feto y comportamientos en la interacción con él que se relacionan con las representaciones cognitivas que la madre tiene de sí misma como cuidadora, es decir con su identidad materna. Grimalt \& Heresi (2012) lo entienden como la relación de apego de una gestante y su hijo que inicia durante el embarazo con las representaciones o imágenes internas que esta hace de su hijo. 
El estilo vincular de la madre, es quizá, el elemento más importante de este proceso, pero también lo son su salud, la estabilidad emocional y afectiva, sus hábitos de cuidado y las circunstancias generales que rodean la experiencia de gestación, dado que las evidencias muestran que, cuando la salud del embarazo está alterada o presenta signos de riesgo importantes, el proceso de vinculación puede verse seriamente afectado (Alhusen, 2008; Doan \& Zimerman, 2008; Bouchard, 2011; Mehran, Simbar, Shams, Ramezani-Tehrani \& Nasiri, 2013).

Por otra parte, el vínculo que genera bienestar entre el padre y la madre es llamado vínculo parental, el cual es definido como el inicio del vínculo prenatal que nace a partir de las imágenes internas que los padres van construyendo sobre su hijo a medida que se van convirtiendo en padres durante el avance del embarazo (Grimalt \& Heresi, 2012). En este proceso ocurren cambios en la mujer y en la relación de pareja, donde la identidad y rol de la futura madre, sufren importantes transformaciones.

En resumen, el vínculo prenatal es el grado en que las mujeres tienen conductas que generan afiliación e interacción con su hijo, proporcionando una sensación subjetiva de amor por el niño que está próximo a nacer. Esta es la primera relación importante para el bebé y ha sido fuertemente asociada con la relación madre-hijo después del nacimiento (Eswi \& Khalil, 2012; Bouchard, 2011; Alhusen, 2008).

El vínculo prenatal tiene tres componentes, el cognitivo, que se evidencia en la capacidad de fantasear, en las representaciones maternas sobre el feto y en la capacidad de otorgar características físicas y psicológicas e intenciones al bebé por nacer; el emocional, que se expresa en la capacidad de ser sensible, dar de sí misma, interactuar con el feto y preocuparse por él, por esto se entiende en términos de intensidad o preocupación que tiene la madre por el feto y calidad o sentimientos hacia él; y finalmente, el comportamental, que se refiere a la asunción de roles y prácticas de autocuidado como comer bien, abstenerse de consumir sustancias dañinas, preparar el entorno del niño en el hogar (Doan \& Zimerman, 2008). Estos tres componentes se realizan en el compromiso que tienen los padres hacia el bebé por nacer, además 
aumenta durante el tiempo de gestación, siendo más fuerte durante el último trimestre (Laxton-Kane, Slade, 2002; Bielawska-Batorowicza \& Siddiqui, 2008; Doan \& Zimerman, 2008; Eswi \& Khalil, 2012).

A su vez, el vínculo prenatal se desarrolla a través de tres canales de comunicación: fisiológico, conductual y simpático. El diálogo que se produce, inicia en una etapa temprana del desarrollo intrauterino, cuando la madre siente los movimientos fetales, y responde a estos. Con ello se produce una respuesta hormonal hacia el embrión por el impacto psicológico que se genera en la madre con este encuentro (Fernández, 2005). Respecto al canal fisiológico, los estados emocionales de la madre, se traducen en secreciones y condiciones fisiológicas que traspasan la barrera placentaria; por ejemplo, en nuevos estudios como los de Monk et al. (2000), se ha observado que el estrés y la ansiedad que experimenta la madre durante el embarazo, produce una secreción anormal de sustancias que se transmiten al feto por medio de la placenta generando agitación y taquicardia en el feto. También Lieberman (1970) en su estudio encontró que un niño intrauterino aumenta su ritmo cardíaco y movimientos fetales cada vez que su madre piensa en fumar cigarrillo. Según Little y Hepper (1995), cuando la madre experimenta ansiedad, es decir, genera un cambio psicológico, este se traduce a uno fisiológico que se transmite al bebé alterando su comportamiento fetal.

Con relación al canal conductual, por ejemplo, Clements (1977) demostró que los niños patean cuando se sienten incómodos, ansiosos o asustados. La madre se comunica de manera conductual con su bebé frotándose el vientre para tranquilizarlos ( $L u$ y Halfon, 2003).

Ahora bien, en el canal simpático varios autores plantean la existencia de una memoria precoz antes del nacimiento, también llamada memoria celular o inconsciente. Fernández (2005) hace referencia a la naturaleza innata de las competencias del recién nacido, entendidas como potencial ontogenético.

Existen múltiples canales de comunicación entre la madre y el niño intrauterino, a medida que avanza el desarrollo, los movimientos feta- 
les pasan de ser difusos y generales, a ser más específicos y sincronizados. Por lo tanto, el niño intrauterino es sensible a su entorno, explora su medio ambiente y percibe los movimientos tanto del cuerpo materno como el propio, además, posee alta capacidad discriminativa, pues asimila y se acomoda sintiendo e integrando información en forma de impresiones. El feto y la madre sincronizan sus interacciones; Brazelton (1982) encontró que en los últimos meses de embarazo algunas gestantes reconocían pautas de comportamiento fetal y eran capaces de responder a ellas, dado que el niño intrauterino desarrolla planos sensoperceptivos, motrices y comunicacionales que le permite dar respuesta a la estimulación sonora externa, a la estimulación vibroacústica, a la voz materna y respuesta discriminativa a estímulos lingüísticos y musicales.

Desde esta perspectiva, el feto se estructura de acuerdo a su código genético y por su base bioquímica se comunica con el organismo de la madre; es decir, hay un intercambio biológico (a través de la placenta y la sangre que transmiten los nutrientes necesarios para el desarrollo del feto) y psíquico (las alteraciones emocionales de la madre tienen consecuencias en su organismo, por lo tanto en el feto) (Relier, 1994).

La madre y el hijo intrauterino establecen un diálogo emocional a través de los sistemas neurohormonales compartiendo un diálogo emocional que «suele iniciar la madre al procesar una acción o pensamiento, su cerebro se convierte instantáneamente en una emoción y orienta su organismo para que produzca un conjunto adecuado de respuestas» (Monk et al., 200o, p.26). Entonces no se puede hacer una distinción entre lo biológico y lo afectivo pues las emociones que vivan las madres van a desencadenar secreciones hormonales que van a modificar el medio biológico del feto, por esto el apego es una realidad biopsíquica en interacción.

El cerebro se desarrolla por dos mecanismos, centrífugo (determinado por herencia genética) y centrípeto (determinado por estímulos exteriores) esto habla de una relación bioafectiva o biopsicológica pues el deseo de tener un hijo en la madre determinará una actitud preven- 
tiva, es decir, condiciona su estado fisiológico (Manger, 1995), o sus cuidados de salud materno-fetal (Fessler, 2003). Entre las 6 y 7 semanas de gestación se empiezan a desarrollar los sistemas sensoriales diferenciados, es decir, ya hay percepción (aunque es un sistema inmaduro) es decir, hay posibilidad de intercambio donde el embrión reacciona a los estímulos maternos (Martinez y Villarraga, 2001). Desde la perspectiva afectiva, que si bien, no puede separarse de la biológica, puede afirmarse que desde la vida intrauterina hay una interacción entre las señales que la madre percibe de su hijo y la forma en que éste las corresponde (Partis, 2000).

Por su parte, la realidad social se fundamenta en la realidad sociocultural de la gestante «el diálogo afectivo entre la madre y el futuro niño se amplía por medio del diálogo entre la madre y el padre, y ésta con el ambiente social» (Fernández 2005, p. 29); las situaciones ambientales de la madre afectan al niño intrauterino pues pueden promover conductas desadaptativas o por el contrario puede promocionar la salud (Morales, Kornblit, Páez \& Asún, 2002).

Respecto a la evolución del vínculo prenatal, Leifer (1977) afirma que el apego prenatal se «desarrolla de manera secuencial y ordenada durante todo el curso del embarazo» (p. 21), siendo el primer trimestre donde se observan niveles relativamente bajos de unión prenatal, que aumentan, progresando a la conexión que se evidencia en conductas como hablar con el feto, buscarle nombres, atribuirle comportamientos en el segundo y tercer trimestre.

El conjunto de representaciones que la madre hace de su hijo está fuertemente ligado a las experiencias tempranas de apego de su propia infancia. Dichas representaciones están relacionadas con la fantasía, y las expectativas sobre el bebé, las cuales se satisfacen, en alguna medida, con el nacimiento y están estrechamente vinculadas con la representación que la propia madre posee de su identidad materna y el estilo vincular que desarrolló desde su infancia. Stern (1995) afirma que las representaciones mentales maternas se reactivan y funcionan en el desarrollo del embarazo, en especial durante el segundo trimes- 
tre, cuando el bebé se reconoce como un ser más real, esto implica una preparación psicológica de la madre para asumir el rol materno.

Con relación a la caracterización de los tipos o formas de apego prenatal, Grimalt \& Heresi (2012) identifican tres subtipos: mujer con vínculo seguro, mujer con vínculo inseguro ambivalente y mujer con vínculo inseguro evitativo.

Una mujer con estilo vincular seguro, durante el embarazo presenta una conexión intensa con su hijo, evidente en las emociones y percepciones positivas del bebé y de la experiencia de embarazo; realiza descripciones positivas sobre sus movimientos y su personalidad. A partir de esto, es posible que la madre construya una confianza en sí misma y en los otros, tanto en su proceso de gestación, como de parto. La configuración del rol materno es el resultado de las características propias y las representaciones creadas sobre su bebé puesto que, «organiza la experiencia de embarazo con relación a su figura de apego principal y se define a sí misma como madre con relación a su propia figura materna» (Grimalt \& Heresi, 2012, p.243).

Por otro lado, la mujer con estilo vincular inseguro ambivalente no relaciona los movimientos fetales con las descripciones que hace de su hijo, sino que se refiere a este a partir de ideales. Esto se debe a que su representación está influenciada por miedos que generan en ella inseguridad, por lo tanto se mantienen los pensamientos pesimistas sobre el buen desarrollo del embarazo, lo que la hace buscar apoyo y protección. Por consiguiente, el proceso de parto reafirma la inseguridad en sí misma, generando temores de no dar lo mejor como madre y no brindar un adecuado cuidado a su hijo (Grimalt \&Heresi, 2012).

Finalmente, la preocupación de una mujer con estilo vincular inseguro evitativo es, principalmente, la de trasmitir estos estados afectivos a su bebé. Tal como lo enuncian los autores «se resiste a tener representaciones de él por miedo a que estas no correspondan con la realidad» (p. 243). La tendencia en este estilo es que dicha mujer pretende construir una conexión emocional como forma de apego, sin pro- 
yectarse en su rol materno, buscando modificar las pautas maternas recibidas enfocándose en las experiencias negativas.

Los diversos tipos de vínculo están determinados por varios factores que influyen en dicho apego, entre ellos, la historia de apego de la madre; esto es, la percepción positiva o no de la relación con los padres y el estilo de apego que se desarrolla a través de experiencias de la infancia que puede influir en la capacidad para vincularse con el feto (Doan \& Zimerman, 2008); también son muy relevantes la edad de la materna, pues las madres con mayor edad tienden a tener más pensamientos positivos con respecto a su bebé (Eswi \& Khalil, 2012; Brandon, 2006); los antecedentes obstétricos que pueden predisponer a la madre respecto al nuevo embarazo: pérdida fetal anterior, antecedentes de embarazos de alto riesgo y antecedentes de embarazos no deseados (Laxton-Kane \& Slade, 2002; Mehran et al. 2013); el estado emocional de la madre, dado que altos niveles de depresión y ansiedad pueden irrumpir en el apego prenatal afectando su calidad (Alhusen, 2008), la propia imagen materna, la calidad de la relación con los demás miembros de su familia, el apoyo percibido y la adaptación de la familia al embarazo (Mehran et al., 2013; Laxton-Kane \& Slade, 2002), así como la calidad de atención percibida que recibe la madre de los sistemas de salud (Laxton-Kane \& Slade, 2002).

\section{Estilo de Vida, Cuidados en Salud Gestacional y el Vínculo Prenatal}

El concepto 'estilo de vida' aparece en las Ciencias Sociales, y muy específicamente en la psicología de la salud, como una categoría de análisis que pretende integrar de manera coherente las explicaciones desarticuladas acerca de cómo o por qué las personas se comportan de determinada manera respecto al cuidado y mantenimiento de su salud e incluso, cómo afrontan la aparición de la enfermedad. En términos generales, según Nutbeam (1996, citado por Pardo y Núñez, 2008), se comprende como «la manera general de vivir, basada en la interacción entre las condiciones de vida, en un sentido más amplio, y las pautas individuales de conducta, determinada por factores socioculturales y 
características individuales» (p. 383). El estilo de vida de las personas, si es saludable, va a permitir que lleven una vida sana y armónica, en cambio, si es perjudicial puede generar riesgo de enfermedades o muerte.

Según la evidencia encontrada, entre las prácticas de cuidado de las gestantes que permiten desarrollar un estilo de vida saludable para ellas y sus bebés, que pueden cambiar la percepción que estas tienen del riesgo obstétrico y favorecer la relación del binomio y, por tanto, el vínculo prenatal, están: la estimulación prenatal, que son las acciones que la madre realiza para estimular a su bebé antes del nacimiento; la actividad física y descanso, que deben ser periódicos para contribuir al bienestar físico de la madre y el feto, mejorando el sueño, circulación, tono muscular y ayudando a evitar el excesivo aumento de peso; la higiene y cuidados personales, que hacen que la gestante pueda mantenerse limpia y cómoda, con el fin de evitar posibles infecciones en ella y su hijo; la alimentación, dado que en esta etapa aumentan los requerimientos nutricionales, por lo tanto, es importante garantizar que ambos reciban los nutrientes necesarios para estar saludables; los sistemas de apoyo social donde la madre debe contar con una red de acompañamiento informacional y emocional siempre disponible y evitar el consumo de sustancias no beneficiosas que perjudiquen tanto al bebé como la salud materna (Granados \& González, 2011).

Por su parte, la depresión en la madre, vista como riesgo psicosocial, puede afectar el desarrollo del embarazo y del vínculo, entre otras razones, porque contribuyen a la producción de hormonas como la noradrenalina y cortisol que repercuten directamente en el feto. A propósito del proceso de vinculación afectiva, Flykt, Kanninen y Sinkonnen (2010) afirman que el impacto de la depresión perinatal parece ser más severo que el de la depresión postnatal, ya que las madres que sufrieron la primera fueron menos sensibles con sus hijos que las que sufrieron de depresión postparto. Así es cómo Hart y McMahon (Alhusen, 2008) al examinar el impacto de la depresión y la ansiedad en el apego prenatal materno, encontraron en su estudio, que las mujeres caracte- 
rizadas por baja calidad del apego fetal informaron significativamente mayores niveles de depresión y ansiedad.

Estas prácticas pueden verse afectadas por las condiciones sociales y económicas de las madres. De acuerdo con Cano et al. (2005), el número de ecografías, las pruebas invasivas, la planificación del embarazo y el consumo de tabaco están asociados al nivel socioeconómico de la gestante. Además, concluyen que la calidad de los cuidados que tenga la madre en este período está relacionada con la planeación del embarazo y la escolaridad, entre otros aspectos sociodemográficos.

En síntesis, como lo afirman Benitzhak \& Verny (2004), existen, además, algunos factores psicosociales que pueden afectar el embarazo y dentro de él, el desarrollo de un vínculo prenatal sólido y seguro que garantice los mejores cuidados de la salud gestacional: entre otros se encuentran las condiciones de desigualdad y vulnerabilidad por aspectos socioeconómicos, de salubridad, políticos y culturales y el estrés materno que afecta tanto a la madre que lo experimenta, como al bebé en desarrollo. Esta afectación impacta a partir de tres mecanismos a saber: reducción de flujo sanguíneo del feto, transporte placentario de hormonas maternas y liberación de corticotropina (CRH) (Mulder, Robles de Medina, Huizink, Van den Bergh, Buitelaar \&Visser, 2002, citados en Pinto, Aguilar \& Gómez, 2010).

Ahora bien, la calidad de atención en salud durante la gestación, puede ser un factor atenuante a estas condiciones psicosociales. La psico-educación y en general las prácticas de atención y acompañamiento en salud que reciben las madres en los centros de atención, son de vital importancia para potencializar los cambios de hábitos en salud y el aumento de las conductas de cuidado gestacional; de acuerdo con Laxton-Kane \& Slade (2002), la atención prenatal tiene dos áreas de enfoque: en primer lugar, las que son responsabilidad de la madre y se refiere a las conductas de autocuidado, definido como fortalecimiento de la capacidad para decidir sobre su persona de forma integral, en aspectos de auto-descubrimiento, autoestima, salud, sexualidad y empoderamiento como protagonista de su propio desarrollo. 
La deficiencia en prácticas de autocuidado en gestantes pueden tener como consecuencia el deterioro de su salud y la de su bebé, lo que genera un aumento en la morbimortalidad materna (Pastor, Suya, Vásquez, Zavala \& Ramírez, 2006). En segundo lugar, las recibidas por los entes en salud, que tienen el objetivo de identificar factores de riesgo y enfermedades que pueden ser detectadas durante el desarrollo del embarazo, para así llevar a cabo acciones preventivas que beneficien la salud de la materna y su bebé (Herrera, 2002).

Existen hipótesis respecto a que el apego prenatal es un factor que está ligado a la calidad en los cuidados (Cano et al., 2005), por ejemplo, los bajos niveles de unión prenatal pueden estar relacionados con diversas formas de abuso fetal, que puede ser pasivo, por omisiones en el cuidado y atención al embarazo, como no asistir a controles, no generar cambios asociados al nuevo estado, para potenciar las condiciones de desarrollo del feto, entre otras; o activos, como por ejemplo, la ingesta de drogas, consumo de alcohol, tabaquismo, trastornos de la alimentación o la autoagresión (como pegarse en el estómago). Sin embargo, es necesario desarrollar más investigaciones sobre el tema, puesto que podría ser útil para disminuir el riesgo fetal (Laxton-Kane \& Slade, 2002).

Por su parte, el riesgo obstétrico entendido como la probabilidad que tiene la madre o él bebe en gestación, o ambos, de sufrir alguna enfermedad y/o la muerte durante el embarazo, puede ser otro factor significativo para el proceso vincular en la etapa gestacional.

White, McCorry, Scott-Heyes, Dempster y Manderson (2008) afirman que cuando existe riesgo obstétrico, la percepción que la gestante tiene de este, influye en la estrategia de afrontamiento utilizada, si es positiva, se predicen mayores niveles de apego prenatal, en cuanto calidad e intensidad del vínculo. Por esta razón, el apego prenatal es fundamental para la adaptación de la madre y mejoramiento de las conductas de salud materna (Mehran et al., 2013). Otros autores afirman que cuando hay riesgo o posible riesgo en el período de gestación, la madre debe adaptar su comportamiento y acrecentar sus cuidados 
y esto puede conducirla a una interacción más intensa aumentando la unión prenatal, sin embargo, lo más común es que el riesgo asociado disminuya este apego (Laxton-Kane \& Slade, 2002).

En contradicción a lo expuesto, Canella (Brandon, 2006) reporta resultados donde el apego prenatal no se ve afectado por el riesgo, sin importar su tipo y su nivel, puesto que no es lo suficientemente fuerte para generar este efecto. De igual manera, Brandon (2006) describe que las mujeres reportan un fuerte apego independientemente del riesgo que su bebé representa para sus vidas. Este autor afirma que no hay relaciones significativas entre riesgo obstétrico y vínculo prenatal y tampoco entre este último y las representaciones maternas. Igualmente Bielawska-Batorowicza \& Siddiqui (2008) afirman que no hay relación entre la diabetes gestacional y el apego de la gestante. White et al. (2008) tampoco encontró una relación significativa entre riesgos médicos y apego prenatal, pero dicha condición médica sí influye en la estrategia de afrontamiento de las madres ante dicha situación.

Por el contrario, Muller (1990, citado Laxton- Kane \& Slade, 2002) sostiene que existe una correlación significativa entre apego prenatal y los embarazos de alto riesgo, puesto que el primero puede verse afectado por la complicación médica existente, pues lleva a la madre a distanciarse del feto para evitar cualquier peligro o sufrimiento por la posible pérdida. Se evidencia entonces la existente contradicción frente a cómo el alto riesgo obstétrico en la madre pueda o no afectar el vínculo prenatal y postnatal y cómo se asocia dicho vínculo con los hábitos de salud y los cuidados maternos, especialmente en condiciones clínicas previas de riesgo; lo cual demuestra la necesidad de mayor investigación respecto a la asociación entre dichas variables.

Por su parte, los padres o compañeros deben hacer ciertos cambios para favorecer el proceso de gestación y la llegada del bebé, así el apego es esencial para el bienestar del infante, además la relación que se genere entre la madre y el padre va a generar impacto en el bienestar de la gestante y en su participación en prácticas de salud como recibir atención prenatal, tener una dieta nutritiva y practicar actividad física 
regularmente. Además, va a ser importante en el desarrollo del apego durante la infancia, porque de acuerdo a esta relación diádica, el niño durante su crecimiento, desarrollará una serie de representaciones mentales sobre su cuidador y sobre sí mismo, que va a determinar su identidad y los prototipos de relación significativa en la niñez y la adolescencia, como extensión y generalización de estas representaciones iniciales (Laxton-Kane \& Slade, 2002; Alhusen, 2008; Grimalt \& Heresi, 2012).

Con base en todo lo anterior, la atención prenatal implica el apoyo directo a las madres embarazadas respecto a los cambios de hábitos y el incremento en sus rutinas de cuidado que acrecienten su salud y disminuyan los riesgos de las gestantes. Con relación a estos aprendizajes, los diferentes comportamientos y conductas que tiene el ser humano a lo largo de su vida, son producto de una serie de pautas aprendidas que nos permiten adaptarnos o responder a las demandas del medio. Sin embargo, el aprendizaje no es referido únicamente a la adquisición de acciones positivas sino que el ser humano, puede aprender acciones saludables y no saludables (Perea, 2004). Además, los procesos de aprendizaje están atravesados por procesos afectivos y emocionales que hacen parte de la trama narrativa de la vida durante todo su ciclo vital y, muy especialmente, estos elementos afectivos se activan en momentos vitales, como puede ser la experiencia de estar embarazadas, y remueven recuerdos y sentimientos asociados con las conductas de cuidado y la calidad afectiva que demanda esta circunstancia vital.

En este sentido, en los programas de atención prenatal, el papel de los psicólogos y los profesionales de la salud en general, buscará promover estilos de vida saludables por medio del aprendizaje, ayudando a las madres a desarrollar hábitos y rutinas vinculados a aspectos tales como la alimentación, ejercicio físico, higiene, entre otras (Libertad, 2003); de ahí la importancia de la educación en salud para incentivar en las pacientes cambios en sus vidas que la favorezcan. Este proceso se ha caracterizado por pedagogías de aprendizaje tradicional basados en la información y la instrucción y, si bien, son metodologías muy im- 
portantes que aportan a la adquisición de nuevas conductas, poco puede afirmarse respecto a su potencia para generar cambios altamente significativos o que impliquen grandes esfuerzos.

Los organismos internacionales como OMS/OPS reconocen que: «el modelo medicalista continúa influenciando en forma desmedida un enfoque por enfermedad y riesgo, menospreciando la influencia de las condiciones y los determinantes sociales» (Peñaranda-Correa et al., 2011, p. 998). De igual manera «la pedagogía tradicional denominada por algunos como pedagogía "instruccional", configuran la base pedagógica predominante de la educación para la salud» (998); sin embargo, cuando las prácticas pedagógicas permiten escenarios de encuentros más dialógicos y conversacionales donde las subjetividades e intersubjetividades emergen en un escenario neutral y generador de confianza intergrupal, les permite a los participantes identificarse como sujetos en sus propias vivencias, con historia, sentimientos, conocimientos y expectativas propias e individuales, la experiencia educativa puede permitir reflexiones y comprensiones nuevas que pueden ser mucho más efectivas para el cambio.

En este contexto, la educación para la salud (EPS) es entendida como «un proceso de educación permanente que se inicia en los primeros años de la infancia orientado hacia el conocimiento de sí mismo en todas sus dimensiones tanto individuales como sociales, y también del ambiente que le rodea en su doble dimensión, ecológica y social con objeto de poder tener una vida sana y participar en la salud colectiva» (Perea, 2004, p. 45), por tanto, busca promover estilos de vida saludables para elevar el nivel de bienestar bio-psico-social. Su propósito entonces va más allá de instruir en una serie de comportamientos y modificar los contextos en donde se desarrollan. Cómo señala Peñaranda-Correa et al. (2011):

Es posible avanzar más allá de una educación centrada en la información y el desarrollo de unos contenidos preestablecidos por los agentes hacia una educación que considera la pertinencia de considerar al ser humano como un ser consciente, crítico, autónomo y libre; un ser trascendente, 
transformador, que crea y recrea, que conoce y comprende y que está abierto a la realidad (p. 1004).

De igual manera Freire (Peñaranda-Correa et al., 2011) argumenta que cada ser humano debe ser considerado «un ser histórico, social y cultural, que no está solo y, por lo tanto, está en relación con el mundo y con otros» (p.1005). Desde esta perspectiva es posible que la experiencia educativa posibilite un espacio donde las madres gestantes pongan en consideración sus vivencias y significados afectivos sobre la nueva maternidad e identifiquen los propios determinantes históricos, biográficos, afectivos y culturales sobre dicha vivencia a partir de su historia relacional.

La ejecución de conductas o hábitos saludables aprendidos implican una responsabilidad individual, para que la información suministrada y 'aprendida' se evidencie en hechos. Así, la autoestima y el autoconcepto son determinantes para la iniciación o mantenimientos de dichos actos donde están fuertemente implicados los procesos afectivos.

En primer lugar, la autoestima, entendida como la valoración de sí mismo y el 'cómo le gustaría ser'; cuando la percepción es positiva, la persona comprenderá que es importante realizar, adoptar o cambiar rutinas a favor de su propio ser y acorde a su imagen ideal. Es así como el sentido de valía propia y de cuidado de sí de la madre gestante influencia la manera como cuida de sí misma y del bebé en gestación y los cambios de hábitos y conductas que conlleva su estado en este período. El autoconcepto, hace referencia a la imagen que se tiene de uno mismo y al igual que el anterior, si es positivo se convierte en un determinante para mantener el equilibrio emocional y la seguridad en sí mismo, y le permiten a la persona procesar la información y hacer algo acorde a ella y en pro de su salud. En tal caso, el autoconcepto y la identidad materna es susceptible de reflexionarse e incrementarse a partir de experiencias formativas que permitan a las gestantes que participan en los programas de promoción de la salud gestacional y el cuidado del embarazo, repensarse en su rol de madres y lograr una mayor conciencia de la importancia de su salud y bienestar emocional 
a la luz de su experiencia subjetiva y su realidad psicosocial y cultural, así como de su manera particular de relacionarse consigo misma, con los otros y con su bebé por nacer, en un proceso progresivo de empoderamiento materno desde sus propios recursos afectivos, su reflexión como seres humanos históricos y protagonistas de su realidad personal e interpersonal.

Esta reflexión, para que alcance su mayor potencia, deberá pasar por procesos de resignificación emocional, entendida como un proceso de análisis que implica responder a ciertas situaciones y a un contexto, a través de la reflexión para interpretar desde otro punto de vista las experiencias vividas, es decir, hacer visibles las emociones, sentimientos y pensamientos que orientan a las acciones y tomar consciencia de estos, para intentar justificar y comprender la situación o contexto con el fin de obtener un aprendizaje, descubrir los motivos y conductas de una forma más racional y de encontrar una mayor precisión y asertividad en el momento de responder a situaciones similares que puedan presentarse en un futuro (Cruz, 2008).

Por consiguiente, al resignificar la propia historia relacional, los acontecimientos o sucesos de la vida cotidiana relacionados con los vínculos más significativos a lo largo de la vida, se realiza una nueva lectura de la vida, que busca ser mejor o superior a esa primera respuesta que se tuvo en el momento de enfrentarse a la situación. Al instaurar este proceso dentro de la vida, se abre la posibilidad de conocerse profundamente, de responder de una forma más favorable a sucesos complejos o adversos que se puedan presentar y a generar estrategias y herramientas adecuadas para las mismas.

Para resignificar un acontecimiento en la vida, no es necesario narrar por segunda vez lo que se hizo, experimentó y sintió en ese momento, pues consiste en repensar e ir más allá, ponerse en situaciones extremas, reflexionar sobre los sentimientos y emociones directamente, las conductas y comportamientos que hicieron parte de la respuesta de cómo afrontar dicha situación. Tal es el caso de las experiencias de relación parental y los vínculos tempranos de las madres gestantes en 
su propia biografía infantil.

Según Berenzon (2003), el proceso de resignificar puede compararse con una traducción, pues es volver a interpretar y construir un acontecimiento para cuestionarse, reflexionar y confrontarse subjetivamente con la forma en que se afrontó el mismo; en este sentido se traen cosas del pasado (recuerdos, sentimientos, pensamientos y emociones) que permiten reinterpretar el contexto y así lograr una reelaboración que adquiere características más favorables y positivas, que a su vez sirven de herramientas para próximas experiencias que la persona pueda afrontar, tal como ocurre en la experiencia de convertirse en madre y su correspondencia con sentimientos de confianza en su propia capacidad de 'maternar' en el nuevo bebé, de cuidar y proveer la estabilidad afectiva necesaria para su sano desarrollo.

\section{Conclusiones}

A partir de esta revisión de tema es posible afirmar que la resignificación del vínculo afectivo y el estilo vincular previo a la experiencia de embarazo, dota de valor a la persona, le proporciona una visión más objetiva y racional de las situación con la que se confrontó y ayuda a la resolución de futuras problemáticas que puedan presentarse en cualquier momento, permitiendo transformaciones más significativas respecto al vínculo prenatal con su bebé y el vínculo de apego posterior al nacimiento; sin embargo, es importante denotar que detrás de este proceso, está ligado un sentido de compromiso y responsabilidad personal, pues involucra el sistema de normas, valores y criterios que tenga instaurada la persona a través de su significados y representaciones culturales y familiares.

En este sentido, la sugerencia es la de considerar el abordaje directo por parte de personal entrenado en los equipos de atención primaria en salud y del servicio hospitalario en general que atiende a población materna, en cuanto al vínculo afectivo prenatal como elemento central del autocuidado materno, así como de las condiciones emocionales y la red de apoyo afectivo y social de las madres gestantes, considerados cómo núcleo de las actividades de control prenatal, así como de las ac- 
tividades educativas y de promoción de la salud y el cuidado del embarazo. Abordar estos elementos afectivos y subjetivos es indispensable para posibilitar la generación de condiciones de cambio y mejoramiento en la disposición afectiva de las madres hacia sus hijos, así como de su compromiso para realizar los cambios de hábitos y conductas necesarios para garantizar su sano desarrollo intrauterino y posterior al nacimiento, desde un sentido fenomenológico de mayor conciencia y bienestar personal.

Activar sentimientos de apego seguro a través de actividades reflexivas, lúdicas y de educación experiencial que les permita a las maternas identificar sus propias experiencias tempranas respecto a la relación primaria de apego con sus padres o cuidadores, así como las representaciones sobre su propia maternidad actual, les permite a las gestantes hacerse cargo de su propia historia y darle un nuevo y más fuerte significado a su experiencia actual de maternidad y así contribuir a la consolidación de vínculos afectivos más saludables de amor y cuidado, a través de la responsabilidad que pueden asumir con mayor empoderamiento y autoconfianza de sus cambios de conducta, de su rol cómo cuidadoras y de su propia dimensión afectiva personal e interpersonal. Los aspectos emocionales, afectivos y socio familiares, en especial, la relación de pareja, cuando existe, son elementos de trabajo del equipo de atención en salud definitivos para posibilitar escenarios que ofrezcan mayor garantía de consolidación de diadas madre-hijo más saludables y promotoras del desarrollo. 


\section{Referencias}

Alhusen, J. L. (2008). A literature update on maternal-fetal attachment. Journal of Obstetric, Gynecologic, \& Neonatal Nursing, 37(3), 315-328. doi: 10.1111/j.15526909.2008.00241

Balay, J. (1994). Terapia cognitiva posracionalista. Conversaciones con Vittorio Guidano. [Postrationalist cognitive therapy: Conversations with Vittorio Guidano]. Buenos Aires: Bibulous.

Benitzhak, Y., Verny, T. R. (2004). The nature of stress due to terrorism on pregnant women and their offspring. Journal Of Prenatal And Perinatal Psychology And Health, 19, 65-74. Disponible en https://birthpsychology.com/journals/volume-19-issue-1/nature-stress-due-terrorism-pregnant-women-and-their-offspring

Berenzon, B. (2003). La Resignificación y la Historia. Facultad de filosofía y letras. UNAM. México, DF. Recuperado de https://www.google.com.co/search?q=resignificacion \&newwindow $=1 \& b i w=1366 \& b i h=667 \&$ ei $=$ vws3VcObLNHisASihY HABA\&start $=0 \& s a=N \#$

Bielawska-Batorowicz, E., Siddiqui, A. (2008). A study of prenatal attachment with Swedish and Polish expectant mothers. Journal of Reproductive and Infant Psychology, 36, 373- 387. Recuperado de http://www.ingentaconnect.com/content/routledg/cjri/2008/00000026/00000004/artoooo8

Blair-Gómez, C. (2013). The biological basis of parent-infant attachment: Foundations and implications for further development. Informes Psicológicos, 13(1), 2340. Recuperado de https://revistas.upb.edu.co/index.php/informespsicologicos/issue/view/223

Bouchard, G. (2011). The role of psychosocial variables in prenatal attachment: an examination of moderational effects. Journal of Reproductive and InfantPsychology. 29(3), 1997-207. doi: 10.1080/02646838.2011.592975 
Bowlby, J. (1986). Vínculos afectivos: formación, desarrollo y pérdida. Madrid: Morata.

Bowlby, J. (1989). Una Base segura: Aplicaciones clínicas de una teoría del apego. Buenos Aires: Paidos.

Brandon, A.R. (2006). Maternal and fetal representations, dimensions of personality, and prenatal attachment in women hospitalized with high-risk pregnanacy. (Tesis Doctoral, The University of Texas Southwestern Medical Center at Dallas). Recuperado de https://repositories.tdl.org/utswmedir/bitstream/handle/2152.5/481/ brandonanna.pdf?sequence $=3$

Brazelton, T. B. (1982). Joint regulation of neonate-parent behaviour. En E. Z. Tronick (Ed.), Social interchange in infancy-affect, cognition and communication. Baltimore: University Park Press.

Cano, G., Rodríguez, M., Borrella, C., Pérez, M., Salvador, J. (2005). Desigualdades socioeconómicas relacionadas con el cuidado y el control del embarazo. Gaceta sanitaria, 20(1), 25-30. Recuperado de http://scielo.isciii.es/pdf/gs/v2on1/original2.pdf

Clements, M. (1977). Observations on certain aspects of neonatal behaviour in response to auditory stimuli. Paper presented at the 5th International Congress of Psychosomatic Obstetrics and Gynecology, Roma. Recuperado de http://catalog.nyam. org/cgi-bin/koha/opac-detail.pl?biblionumber=20019

Cruz, D. (2008). Seminario-taller para el fortalecimiento técnico-operativo de los Supervisores de educación especial de la CROSEEE. núm. 3. Documento de referencia, fotocopia, México, Dirección de Educación Especial.

Doan, H., Zimerman, A. (2008). Prenatal Attachment: A Developmental Model. The international Journal prenatal and perinatal psychology and medicine, 20 (1/2), 20- 28. Recuperado de http://www.steigerweg.de/buecher/praenatale_psychologie/ PP_PDF/PP_2O_1-2_Doan_Zimerman.pdf 
Eswi, A., Khalil, A. (2012). Prenatal Attachment and Fetal health locus of control among low risk and high-risk pregnant women. . World Applied Sciences Journal, 18(4), 462-471.Recuperado de http://www.idosi.org/wasj/wasj18(4)12/1.pdf

Herrera, J. (2002). Evaluación del Riesgo Obstétrico en el Cuidado Prenatal. Colombia médica, 33(1), 21-25. Recuperado de http://www.redalyc.org/pdf/283/28333104. pdf

Fernández, L. M. (2005). El vínculo afectivo con el niño por nacer. Salamanca, España: Universidad Pontificia de Salamanca.

Fessler, K. B. (2003). Social outcomes of early childbearing: important considerations for the provision of clinical care. Journal of Midwifery Womens Health, 48 (3), 178-185. doi:10.1016/S1526-9523(03)ooo63-1

Flykt, M., Kanninen, K., Sinkonnen, J. P. (2010). Maternal Depression and Dyadic Interaction: The role of Maternal Attachment Style . Infant and Child Development, 19, 530- 550. Recuperado de. http://onlinelibrary.wiley.com/doi/10.1002/ icd.679/abstract

Guidano, V. (1994). El desarrollo del enfoque evolutivo constructivista en la terapia cognitiva. Santiago de Chile: Instituto de Terapia Cognitiva.

Guidano, V. F. (1999, August). Los procesos del self: continuidad y discontinuidad. En: Conferencia dictada en las Jornadas de psicoterapia cognitiva constructivista posracionalista. . Recuperado de http://www.inteco.cl/articulos/oo8/texto_esp.htm

Guidano, V. (2001). Vittorio Guidano en Chile (Susana Aronsohn F ed.). Recuperado de http://www.posracionalismo.cl/imagenes/vguidano.pdf

Granados, L.M., González Aparicio, A.A. (2011). Prácticas de cuidado que hacen las gestantes adolescentes consigo mismas y con el hijo por nacer. Medunab, 14(1), 9-14. Recuperado de http://revistas.unab.edu.co/index.php?journal=medunab\&page=article\&op=viewArticle\&path\%5B\%5D=1373 
Grimalt, L., Heresi, E. (2012). Estilos de apego y representaciones maternas durante el embarazo. Revista Chilena de Pediatría, 83(3), 239-246, doi:10.4067/So37041062012000300005

Laxton-Kane, M., Slade, P. (2002). The role of maternal prenatal attachment in a woman's experience of pregnancy and implications for the process of care. Journal of reproductive and infant psychology, 20 (4), 253- 266, doi: 10.1080/0264683021000033174

Lecannelier, F. (2009). Evolución y desarrollo del self. Instituto de Terapia Cognitiva INTECO, 4 (3).Recuperado de http://www.inteco.cl/articulos/o12/texto_esp.htm

Lecannelier, F. (2010). La nueva ciencia de los bebés. Apego e intersubjetividad. Recuperado de http://revistagpu.cl/GPU\%201\%20(2010)/ENT\%20Felipe\%20Lecannelier.pdf

Leifer, M. (1977). Psychological changes accompany in pregnancy and motherhood. Genet Psychol Monogr). 95 (1), 55-96. Recuperado de https://www.ncbi.nlm. nih.gov/pubmed/849833

Libertad, M. (2003). Aplicaciones de la psicología en el proceso salud enfermedad. Revista Cubana Salud Pública. 29 (3), 81-275. Recuperado de http://scielo.sld. cu/pdf/rcsp/v29n3/spu12303.pdf

Little, J., Hepper, P. G. (1995). The phychological effects of maternal smoking on fetal movements. International Journal Prenatal and Perinatal Psychology and medicine, 7 (2), 161-167. Recuperado de http://pure.qub.ac.uk/portal/en/publications/ the-psychological-effects-of-maternal-smoking-on-fetal-movements(5c38c374-addb4f31-883f-aogdad74a1d9).html

Lu, M. C., Halfon, N. (2003). Racial and ethnic disparities in birth outcomes: a life-course perspective. Maternal Child Health Journal, 7 (1), 13-30. Recuperado de https://www.ncbi.nlm.nih.gov/pubmed/12710797

Maas, A. J., Vreeswijk, C. M., Cock, E. S., Rijk, C. H. (2012). “Expectant Parents”: 
Study protocol of a longitudinal study concerning prenatal (risk) factors and postnatal infant development, parenting, and parent-infant relationships. BMC Pregnancy and Childbirth, 12(1),46, doi: 10.1186/1471-2393-12-46.

Manger, B. (1995). Birth as metaphor: Childbirth as initiation and transformation. International Journal of Prenatal and Perinatal Psychology and Medicine, 7 (4), 465-474). Recuperado de http://cat.inist.fr/?aModele=afficheN\&cpsidt $=2927530$

Martínez, J. C., Villarraga, S. (2001). Gestación y nacimiento, atención desde una perspectiva humanizante. Comunicación presentada en el quinto curso de actualización de enfermería, celebrado en CAFAM. Recuperado de https:// www.unicafam. edu.co/index.php/escuela-de-enfermeria

Maturana, H. (2008). El Sentido de lo Humano. Ediciones Granica S.A., Santiago, Chile.

Mehran, P., Simbar, M., Shams, J., Ramezani-Tehrani, F., Nasiri, N. (2013). History of perinatal loss and maternal-fetal attachment behaviors. Women and Birth, 26(3), 185-189.Recuperado de https://www.ncbi.nlm.nih.gov/pubmed/23721683.

Monk, C., Fifer, W., Myers, M., Sloan, R., Trien, L., Hurtado, A. (2000). Maternal Stress responses and anxiety during pregnancy: Effects on fetal heart rate. DevelopmentalPsychobiology, 36(1), 67-77, doi:10.1002/(SICI)1098-2302(200001)36:1<67::AID$\mathrm{DEV}_{7}>$ 3.0.CO;2-C

Morales, J, F., Kornblit, A., Páez, D., Asún, D. (2002). Psicología social. Madrid: Prentice Hall.

Oiberman, A. (1998). Padre-bebé: inicios de una relación. La Plata: Editorial de la Universidad de Plata.

Oiberman, A. (2001). Observando a los bebés. Estudio de una técnica de observación de la relación madre-hijo. Buenos Aires: Lugar Editorial.

Pardo, M.P., Nuñez, N.A. (2008). Estilo de vida y salud en la mujer adulta joven. 
Uquichán. 8(2), 266-284. Recuperado de http://aquichan.unisabana.edu.co/index. php/aquichan/article/view/153/292

Partis, M. (2000). Bowlby's attachment theory: implications for health visiting. British Journal of Community Nursing, 5 (10), 499-503. Recuperado de https://www. ncbi.nlm.nih.gov/pubmed/12181518

Pastor, M., Suya, L., Vásquez, I., Zavala, C., Ramírez, T. (2006). Conocimientos y prácticas sobre autocuidado que influyen en la salud de la mujer, durante el embarazo y el puerperio. Revista de la facultad de ciencias médicas, 3(2), 13-18. Recuperado de http://www.bvs.hn/RFCM/pdf/2006/pdf/RFCMVol3-2-2006.pdf

Peñaranda-Correa, F., Torres-Ospina, J. N., Bastidas-Acevedo, M., Escobar-Paucar, G., Arango-Córdoba, A., Pérez-Becerra, F.N. (2011). La praxis como fundamento de una educación para la salud alternativa: estudio de investigación-acción. Comunicação saúde educação, 15 (39), 997-1008. Recuperado de http://www.redalyc.org/ pdf/1801/180121094016.pdf

Perea, R. (2004). Educación para la salud: Reto de nuestro tiempo. España: Editorial Díaz de Santos.

Pinto, MC., Aguilar, O.M., Gómez, JD. (2010). Estrés psicológico materno como posible riesgo prenatal para el desarrollo de dificultades cognoscitivas: caracterización neuropsicológica de una muestra colombiana. Univ. Psychol. 9(3), 749759. Recuperado de http://www.scielo.org.co/scielo.php?script=sci_arttext\&pid $=\mathrm{S} 1657-92672010000300012$.

Relier, J.P. (1994). Ama a tu hijo antes que nazca. Barcelona: Martínez Roca

Safram, J. D., Segal, Z. V. (1994). El proceso interpersonal en la terapia cognitiva. Barcelona: Paidós.

Stern, D. N. (1995). La constelación maternal: La psicoterapia en las relaciones entre padres e hijos. New York: Paidós. 
Valdizan, H. (2003). Antecedentes tempranos y modalidades de vulnerabilidad cognitiva para la depresión. Revista de psiquiatria y salud mental, 4 (2), 43-62. Recuperado de http://www.hhv.gob.pe/revista/2003II/4\%20ANTECEDENTES\%2OTEMPRANOS\%20\%20Y\%20MODALIDADES\%20DE.pdf.

White, O., McCorry, N. K., Scott-Heyes, G., Dempster, M., Manderson, J. (2008). Maternal appraisals of risk, coping and prenatal attachment among women hospitalised with pregnancy complications. Journal of Reproductive and Infant Psychology, 26 (2), 74- 85. Doi: 10.1080/02646830801918455

Young, J. E. (1999). Cognitive therapy for personality disorders: A schema-focused approach (3a ed.). Sarasota, FL, EE.UU: Professional Resource Exchange.

Young, J. E., Rygh, J. L., Weinberger, A. D., Beck, A. T. (2008). Cognitive therapy for depression. In D. H. Barlow (Ed.), Clinical handbook of psychological disorders (4a ed), pp. 250-305. Nueva York: The Guildford Press. 